2014

\title{
Fukushima: A Model Study
}

Melinda Kathryn McKell

University of South Florida

Advisors:

Arcadii Grinshpan, Mathematics and Statistics

Chuck Connor, Geology

Problem Suggested By: Chuck Connor

Follow this and additional works at: https://digitalcommons.usf.edu/ujmm

Part of the Mathematics Commons

UJMM is an open access journal, free to authors and readers, and relies on your support:

Donate Now

\section{Recommended Citation}

McKell, Melinda Kathryn (2014) "Fukushima: A Model Study," Undergraduate Journal of Mathematical Modeling: One + Two: Vol. 6: Iss. 1, Article 6.

DOI: http://dx.doi.org/10.5038/2326-3652.6.1.4862

Available at: https://digitalcommons.usf.edu/ujmm/vol6/iss $1 / 6$ 


\section{Fukushima: A Model Study}

Abstract

This article investigates the radiation dispersion modeling system available to Japanese authorities during the response phase of the Fukushima Dai-Ichi incident.

\section{Keywords}

Fukushima, nuclear incident, dispersion model 


\section{PROBLEM STATEMENT}

The majority of electrical energy in Japan is produced by nuclear power plants, based on driving turbines and generators using high-temperature and high pressure. The plant operates on a cycle that continuously converts water between liquid and steam to move energy from one location in the plant to another. Boiling water reactor nuclear plants are the source of heat for the system. The closed primary loop of water moves heat directly from the core to the steam generator, and cannot leave the containment wall. The steam generator transfers the heat in the primary loop to the water in the secondary loop, keeping the two loops entirely isolated from one another. The steam in the second loop flows to the turbine, which causes the turbine's shaft to rotate, performing mechanical work, similar to a water wheel. The tertiary loop draws outside water into the condenser for cooling purposes. The water supplies within the loops do not mix, but the heat transfers between them (Wickert; 2005). Lack of power and breaches in the loops causes an interruption of cooling, which can led to a core meltdown and escape of radioactive

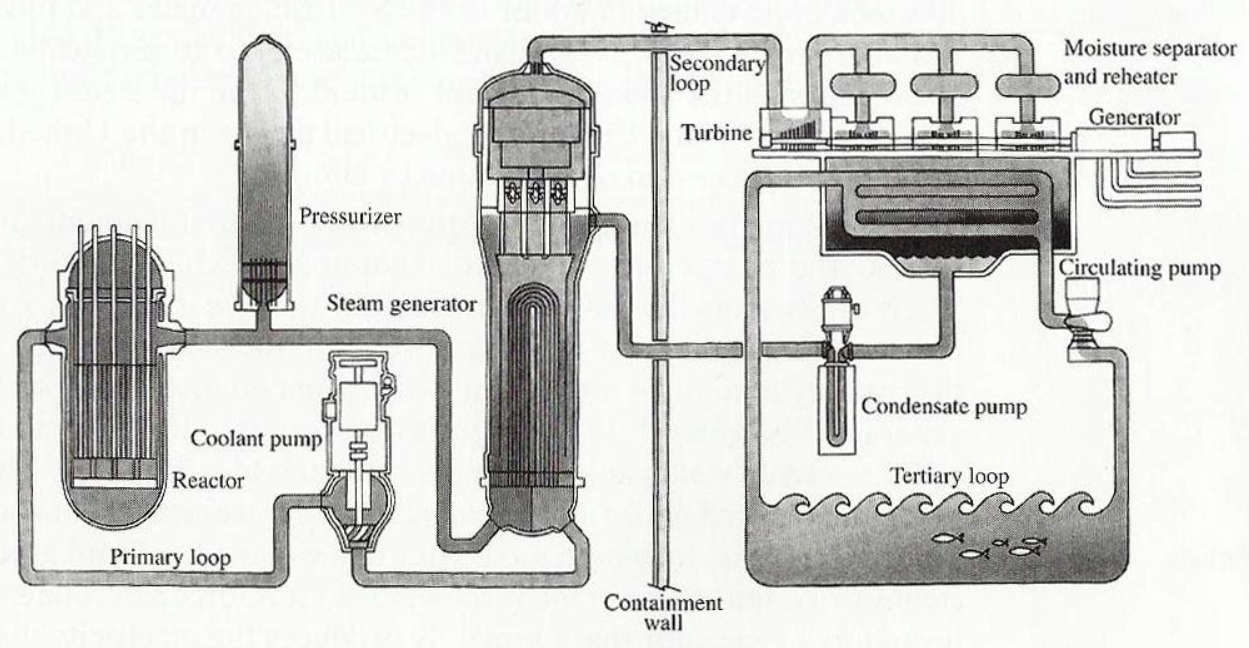

Figure 1: Boiling water nuclear power plant cycle

Keywords: Fukushima, Nuclear incident, Dispersion model 
particles from the containment.

On March 11th, 2011, a tsunami triggered by a magnitude-9.0 earthquake disabled all power to the Fukushima Number 1 Power Plant $\left(37^{\circ} 25^{\prime} 18^{\prime \prime} \mathrm{N}, 141^{\circ} 02^{\prime} 08^{\prime \prime} \mathrm{E}\right)$, destroyed the service road and swept the emergency generator fuel out to sea. Per the protocols governing all 54 of Japan's nuclear power facilities (TEPCO) the plant put itself into automatic shutdown. However, the tsunami cleared the protective sea wall that critically interfered with the cooling systems and flooded the facilities. Hydrogen explosions further damaged structures at the site, releasing a large amount of radioactive material into the environment.

In 1980, Japan's Ministry of Education, Culture, Sports, Science and Technology (MEXT) and Fujitsu collaboratively began to model such disaster situations, and 1985 the System for Prediction of Environmental Emergency Dose Information (SPEEDI), which predicts the spread and effect of radioactive materials in the event of a release from a nuclear facility (Misawa and Nagamori), was debuted. However, on March 11, 2011, when the system began producing models within hours of the massive earthquake and subsequent tsunami, political officials were "unsure how to interpret the results" (Onishi and Fackler). Instead of using the information produced by SPEEDI, on March 14, 2011 a map with rings was released to the public indicating a conservative evacuation zone of $20 \mathrm{~km}$. The zones were expanded on March 25 th to $30 \mathrm{~km}, 10$ days after the biggest spike in radioactivity to date, sending recorded levels up three orders of magnitude. The $20 \mathrm{~km}$ rings did not take into account the mountainous terrain, wind patterns or temperature.

This paper will take a look at the basic underlying math of the computer modeling system that was printing out reports before, during and after the March 11, 2011 disaster. 


\section{MOTIVATION}

The Fukushima incident was raised to Level 7 by the International Atomic Energy Agency (IAEA) on April 12 after Japan's NISA announced their estimate regarding the accumulated amount of the radioactive materials released in the atmosphere (Anzai, Ban and Ozawa). Table 1 compares the amount of Iodine-131 and Caesium-137 released from Fukushima to the April 26, 1986 Chernobyl (Davoine and Bocquet).

\begin{tabular}{|ccc|}
\cline { 2 - 3 } \multicolumn{1}{c|}{} & Fukushima & Chernobyl \\
\hline Iodine 131 & $1.3 \times 10^{17} \mathrm{~Bq}^{1}$ & $1.76 \times 10^{18} \mathrm{~Bq}$ \\
Caesium 137 & $6.1 \times 10^{16} \mathrm{~Bq}$ & $8.5 \times 10^{16} \mathrm{~Bq}$ \\
\hline
\end{tabular}

Table 1: Fukushima versus Chernobyl

Iodine 131 (I-131) is a major uranium, plutonium fission product that has a radioactive decay half-life of about eight day. Caesium 137 (Cs-137) has a half-life of about 30.17 years and is one of the more common fission products by the nuclear fission in nuclear reactors. It is among the most problematic of the short-to-medium-lifetime fission products because it easily moves and spreads in nature due to the high water solubility. Given a sample of a radionuclide, the half-life is the time taken for half the radionuclide's atoms to decay.

Based on the presented values, it is easy to compute decaying times of I-131 and Cs-137 that was released from Fukushima between March 11 and March 15, 2011 (Hsu, Huh and Chan). The following formula is used to compute such decay.

$$
\text { Radiation in } \mathrm{Bq}=\frac{\text { mass in grams }}{\text { atomic mass in } \frac{g}{m o l}}(\text { Avogadro constant }) \frac{\ln 2}{t_{1 / 2}}
$$

\footnotetext{
${ }^{1}$ Becquerel (Bq) is used to express the number of disintegrations of radioactive atoms in a radioactive material over a period of time.
} 
This information can be used later in the dispersion calculation. Some of the constant coefficients used for computations are provided in Table 2.

\begin{tabular}{ll}
\hline Avogadro's constant & $6.02214179 \times 10^{23} \mathrm{~mol}$ \\
I-131 atomic mass & $16,624.4860 \mathrm{~g} / \mathrm{mol}$ \\
Cs-137 atomic mass & $18,208.046913 \mathrm{~g} / \mathrm{mol}$ \\
I-131 decay constant & $\lambda=1.0003538 \times 10^{-6} /$ second \\
Cs-137 decay constant & $\lambda=7.2852 \times 10^{-10} / \mathrm{second}$ \\
\hline
\end{tabular}

Table 2: Computational constant coefficients

Based on the IAEA reports, $1.3 \times 10^{17} B q$ of Iodine-131 was released from Fukushima incident. By using (1), one can determine the approximate mass of I-131 from the incident:

$$
1.3 \times 10^{17}=\frac{\text { mass }}{16,624.4860} 6.02214179 \times 10^{23} \mathrm{~mol}\left(1.0003538 \times 10^{-6}\right)
$$

then, we have

$$
\text { mass }=\left(\frac{\left(1.3 \times 10^{17}\right)(16624.4860)}{6.02214179 \times 10^{23}\left(1.0003538 \times 10^{-6}\right)}\right)=3,587.46 \mathrm{~g}
$$

That is, at time $t=0$, there was $3,587.46 \mathrm{~g}$ of I-131. Now, let $N_{t}$ be the remaining amount of Iodine at time $t$. Noting that Iodine decays exponentially,

$$
N_{t}=N_{0} e^{-\lambda t}
$$

and one can find the required time when the original sample is almost completely gone. That is, the time required for the current I-131 levels to reach the safer level of 2 grams may be computed by solving

$$
2=3587.46 \times e^{-1.0003538 \times 10^{-6} t}
$$

which results in 


$$
t=7,489,403 \text { seconds }=86.68 \text { days. }
$$

Hence, it would take about 87 days for the area to become safe from I-131. Using a similar analysis for the Caesium-137 levels in Table 1, it will take 611.6 years before the Cs-137 levels drop below 2 grams.

\section{MATHEMATICAL DESCRIPTION AND SOLUTION APPROACH}

A dispersion model is a computational procedure for predicting concentrations of a pollutant source. The process of modeling how particles spread in the air utilizes mathematical algorithms and requires data from source characteristics, terrain and meteorology. A variety of models have been used around the world to predict the airborne radiation from Fukushima Number 1 nuclear facility. Japan's model SPEEDI is based on a Gaussian Plume model (Burk; 2005; Larson, Hostetler and Edwards; 2002; Macdonald; 2003).

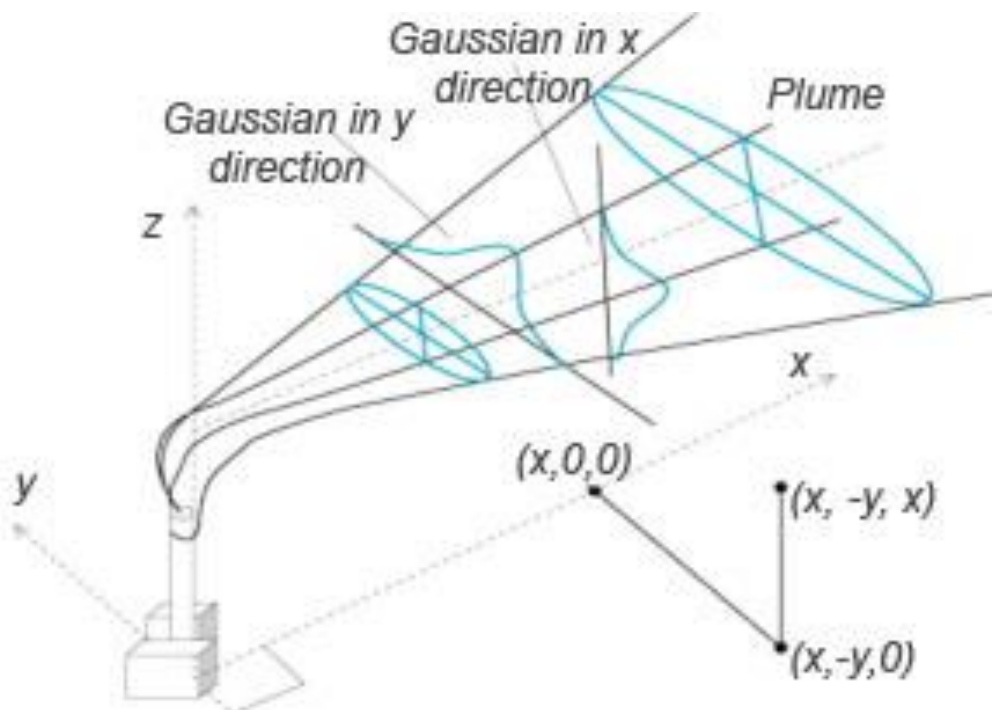

Figure 1: The Gaussian Model (the model is valid up to $10 \mathrm{~km}$ from the source) 
Before taking a look at the Gaussian Plume Model, an understanding of the diffusion relation is necessary. The Diffusion law states that atomic movements generally go from higher to lower concentrations. The rate of diffusion $(J)$ is generally expressed by $J=\frac{F}{R}$, where $F$ is the driving force and $R$ is the resistance to movement of atoms through a lattice and can be written as $R=$ $d \frac{L}{A}$, where $d$ is a constant, $L$ is the distance of atomic movement, and $A$ is the area of diffusion plane. Combining these two equations, results in

$$
J=\frac{1}{d} A\left(\frac{F}{L}\right)=D A\left(\frac{F}{L}\right)
$$

where $D=\frac{1}{d}$ is called the diffusion coefficient. It is defined as the amount of substance diffusing in unit time across a unit area through a unit concentration gradient and is generally expressed in terms of $\mathrm{m}^{2} / \mathrm{sec}$. A gradient on the other hand, is an example of a vector field. That is, function that assigns vectors to points in space.

The diffusion equation is the first step toward deriving the Gaussian Plume Model (Macdonald; 2003). This equation indicates how a continuous cloud of material is dispersing in a turbulent flow.

$$
\frac{\partial C}{\partial t}+U \frac{\partial C}{\partial x}=\frac{\partial}{\partial y}\left(K_{y} \frac{\partial C}{\partial y}\right)+\frac{\partial}{\partial z}\left(K_{z} \frac{\partial C}{\partial z}\right)+S
$$

Different parameters in this equation are $x$ which is along-wind coordinate measured from the source, $y$ is cross-wind coordinate direction, $z$ is vertical coordinate measured from the ground, $C(x, y, z)$ that is discussed in detail later is the mean concentration of diffusing at point $(x, y, z)$ in $\mathrm{kg} / \mathrm{m}^{3}, K_{y}$ and $K_{z}$ are eddy diffusivities in the direction of $y$ - and $z$-axes and are measured in 
terms of $m^{2} / s, U$ is the mean wind velocity along the $x$-axis in $m / s, S$ is the net production or destruction of pollutant in $\mathrm{kg} / \mathrm{m}^{3}$.

The term $\frac{\partial C}{\partial t}+U \frac{\partial C}{\partial x}$ represents the time rate of change and advection of cloud by the mean wind. The term $\frac{\partial}{\partial y}\left(K_{y} \frac{\partial C}{\partial y}\right)+\frac{\partial}{\partial z}\left(K_{z} \frac{\partial C}{\partial z}\right)$ represents turbulent diffusion of material relative to the center of the pollutant cloud. This part also shows that the cloud expands over time. The eddy diffusivities $\left(K_{y}\right.$ and $K_{z}$ ) relate the turbulent fluxes of material to the mean gradients of concentration, that is $\overline{v^{\prime} c^{\prime}}=-K_{y} \frac{\partial C}{\partial y}$ and $\overline{w^{\prime} c^{\prime}}=-K_{z} \frac{\partial C}{\partial z}$. The primed coordinates refer to the turbulent fluctuations, $c(t)=C+c^{\prime}$ and $u(t)=U+u^{\prime}$. If $K_{y}>K_{z}$, the plume takes an elliptical shape.

MacDonald (2003) points out that the Diffusion Equation here is limited in predicting power, because the following assumptions need to be made:

- The pollutant must be passive.

- Molecular and along-wind diffusion are negligible.

- The flow is incompressible.

- The wind velocities and concentrations stochastic component equals to zero.

- The turbulent fluxes are related to the gradients.

- Restricted to an idealized flat terrain.

As for the Gaussian Plume equation, it is given by the following formula

$$
C(x, y, z)=\frac{Q}{4 \pi x \sqrt{K_{y} K_{z}}} \exp \left(\frac{-y^{2}}{4 K_{y}\left(\frac{x}{U}\right)}\right) \exp \left(\frac{-z^{2}}{4 K_{z}\left(\frac{x}{U}\right)}\right)
$$

where $Q$ is the source strength given by 


$$
Q=\iint_{-\infty}^{\infty} C d y d z
$$

for all $x>0$. It works by drawing a cone around the source and making an approximation as Chart 2 illustrates.

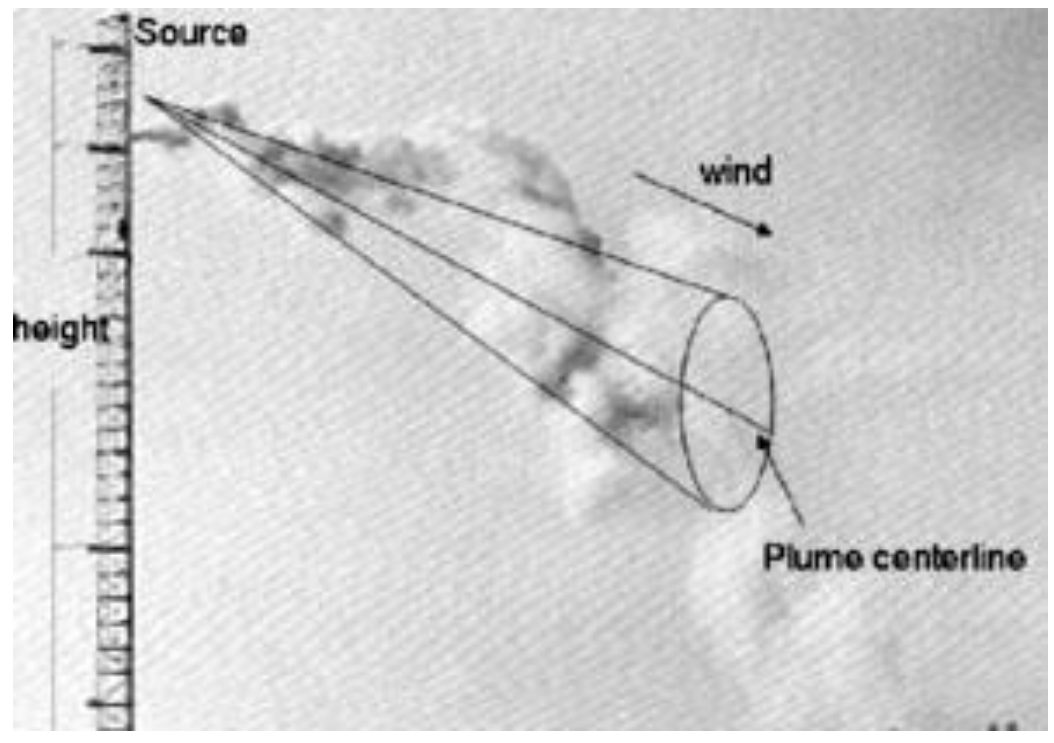

Chart 2: The Gaussian Model (the model is valid up to $10 \mathrm{~km}$ from the source)

However, if the pollution is coming from a continuous source point, released at the origin in a uniform turbulent flow, then this dispersion model can be altered with Gaussian parameters $\sigma_{y}$ and $\sigma_{z}$, the standard deviations on horizontal and vertical deviation respectively, which are commonly used instead of $K_{y}$ and $K_{z}$ (See Chart 3). They are defined as $\sigma_{y}=\sqrt{2 K_{y} \frac{x}{U}}$ and $\sigma_{z}=$ $\sqrt{2 K_{z} \frac{x}{U}}$. After making these substitutions, equation (5) reduces to

$$
C(x, y, z)=\frac{Q}{2 \pi U \sigma_{y} \sigma_{z}}\left\{\exp \left(\frac{-(z-h)^{2}}{2 \sigma_{z}^{2}}\right)+\exp \left(\frac{-(z+h)^{2}}{2 \sigma_{z}^{2}}\right)\right\}\left\{\exp \left(\frac{-(y)^{2}}{2 \sigma_{y}^{2}}\right)\right\}
$$

The plume parameters $\sigma_{y}$ and $\sigma_{z}$ are functions of $x$ determined by turbulence and are influenced by the state of the atmosphere, and $h$ is a constant. In order to relate the state of atmospheric 
convection to simply observable parameters, Pasquill and Gifford (Macdonald) developed a rating system of six stability classes ranging from highly convective [A] to highly stable flow conditions $[\mathrm{F}]$ (See Table 3).

Table 3: Rating system of six stability classes

\begin{tabular}{|c|c|c|c|c|}
\hline Description & $\begin{array}{l}\text { P-G Stability } \\
\text { Class }\end{array}$ & $\begin{array}{l}\text { Time of } \\
\text { Day/Condition }\end{array}$ & $\begin{array}{l}\text { Wind } \\
\text { Speed U }\end{array}$ & $\begin{array}{l}\text { M-O Length } \\
\text { LMO }\end{array}$ \\
\hline Very Unstable & $\mathrm{A}$ & Sunny Day & $<3 \mathrm{~m} / \mathrm{s}$ & $-10 m$ \\
\hline Unstable & $\mathrm{B}$ or $\mathrm{C}$ & & $2-6 \mathrm{~m} / \mathrm{s}$ & $-50 \mathrm{~m}$ \\
\hline Neutral & $\mathrm{D}$ & Cloudy or Windy & $>3-4 \mathrm{~m} / \mathrm{s}$ & $|\mathrm{L}|>100 \mathrm{~m}$ \\
\hline Stable & $\mathrm{E}$ & & $2-4 \mathrm{~m} / \mathrm{s}$ & $+50 \mathrm{~m}$ \\
\hline Very Stable & $\mathrm{F}$ & Clear Night & $<3 \mathrm{~m} / \mathrm{s}$ & $+10 \mathrm{~m}$ \\
\hline
\end{tabular}

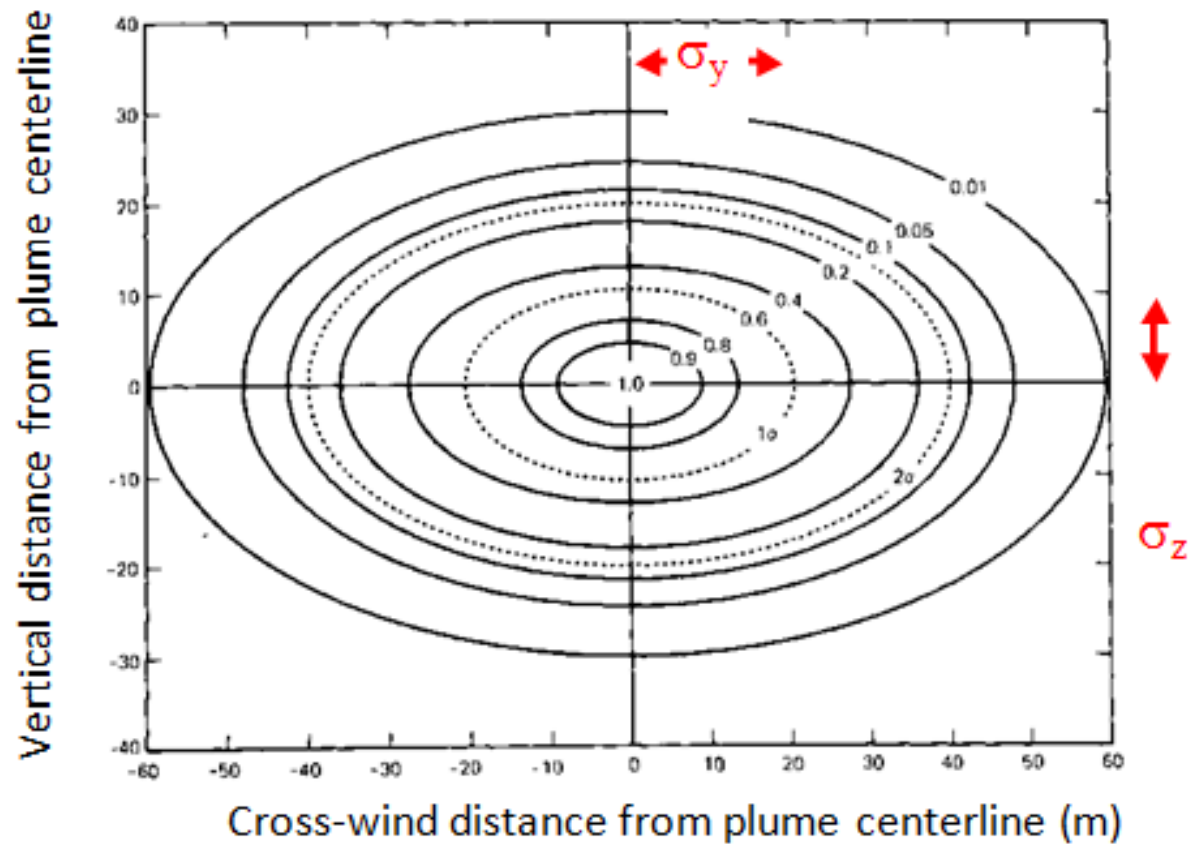

Chart 3: Concentration distribution in a Gaussian plume with $\sigma_{y}=20 \mathrm{~m},=10 \mathrm{~m}$, and centerline concentration $=1.0$ (Theoretical plume has infinite extent in all directions)

The usual Gaussian Plume model is only valid for up to $10 \mathrm{~km}$ when computations are taken by hand. However, Japan's modeling system SPEEDI is able to take the Gaussian Plume 
calculations and extend them to include a variety of source points, more complex atmospheric effects and terrain features. It is accurate for well beyond $10 \mathrm{~km}$. The Gaussian model is the backbone of the sophisticated SPEEDI, which reports emissions in $\mathrm{Bq} / \mathrm{m}^{3}$ (the concentration) by using the MM5 or GEARN codes.

We now move on to interpreting the reports produced by SPEEDI. Consider a point source $20 \mathrm{~m}$ in the air where pollutants are released at a constant rate $\mathrm{Q}(\mathrm{kg} / \mathrm{s})$. The aim is to find the emission rate $Q$ knowing that the wind is blowing continuously in a SSE direction with a speed of $5.4(\mathrm{~m} / \mathrm{s})$. The plume spreads as it moves in the $x$ direction such that the local concentrations $C(x, y, z)$ at any point in space form Gaussian distributions in planes normal to the $x$ direction. Chart 4 provides the vector field map of the atmospheric effects produced by SPEEDI and Chart 5 gives a rough translation of the reports into English.

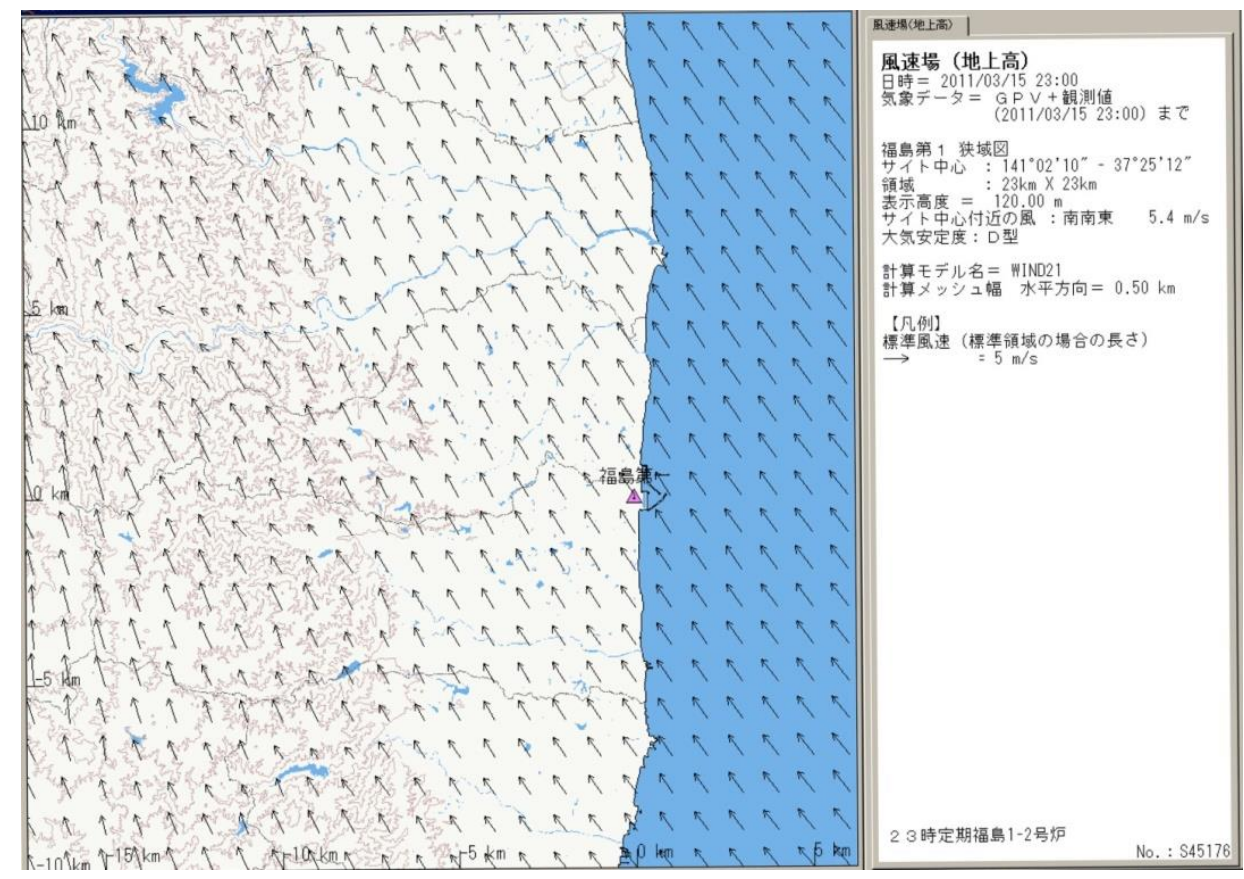

Chart 4: The vector field map of the atmospheric effects of March $15^{\text {th }}$ at $11 \mathrm{PM}$ produced by Japan's SPEEDI modeling system 


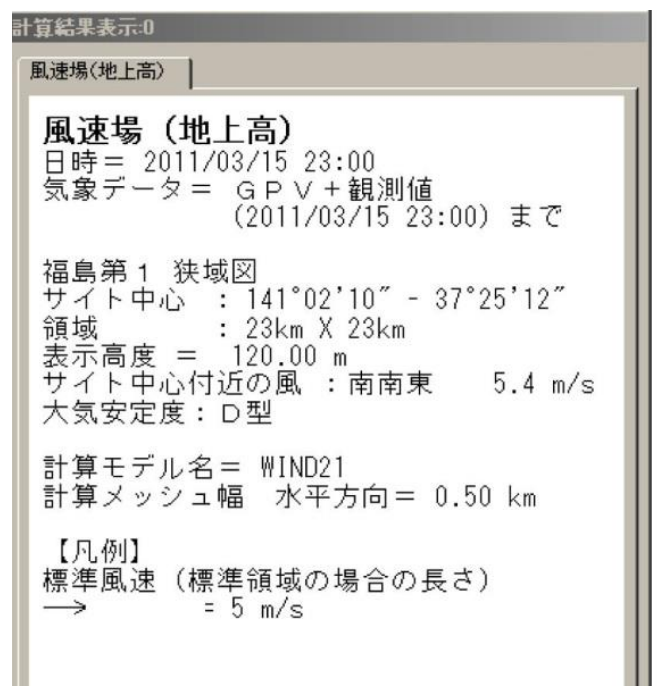

\section{Wind conditions}

Observed weather data for March 15, 2011 until 11PM

Fukushima Number 1 Power Plant Site coordinates: $37^{\circ} 25^{\prime} 12^{\prime \prime} \mathrm{N}, 141^{\circ} 02^{\prime} 10^{\prime \prime} \mathrm{E}$ Radiation grid: $23 \mathrm{~km} \times 23 \mathrm{~km}$ Elevation: $\quad 120.0 \mathrm{~m}$

Wind speed at the site: SSE at $5.4 \mathrm{~m} / \mathrm{s}$ Atmospheric equillibrium measurement : $D$ Calculation code: WIND21

Calculation mesh: $.50 \mathrm{~km}$

Legend:

Standard windspeed (standard area length) $--->=5 \mathrm{~m} / \mathrm{s}$

Chart 5: Rough translation of the vector field map report by SPEEDI

Let us find the values of $\sigma_{z}$ and $\sigma_{y}$ that are required in further computations. The EPA's ISC Model uses $\sigma_{z}=a x^{b}$ to calculate the P-G values where $x$ is in kilometers, $\sigma_{z}$ is in meters, $a$ and $b$ depend on $x$. According to Pasqual and Gifford, when conditions are D (as SPEEDI data states), then $x=1, a=32.093$, and $b=0.81066$. So, $\sigma_{z}=32.093 \times(1)^{0.81066}=32.093$. For the cross-wind distribution, relation $\sigma_{y}=465.11628 \times(\tan \theta)$ is used where $x$ is in kilometers, $\sigma_{z}$ is in meters and $\theta$ is in radians. When the Pasqual Stability category is $\mathrm{D}$, and $c=$ $8.3330, \quad d=0.7238, \quad$ and $\theta=0.017453293(c-d \ln x)=0.145438$ then $\sigma_{y}=$ $465.11628(\tan 0.145438)=1.18064$. 


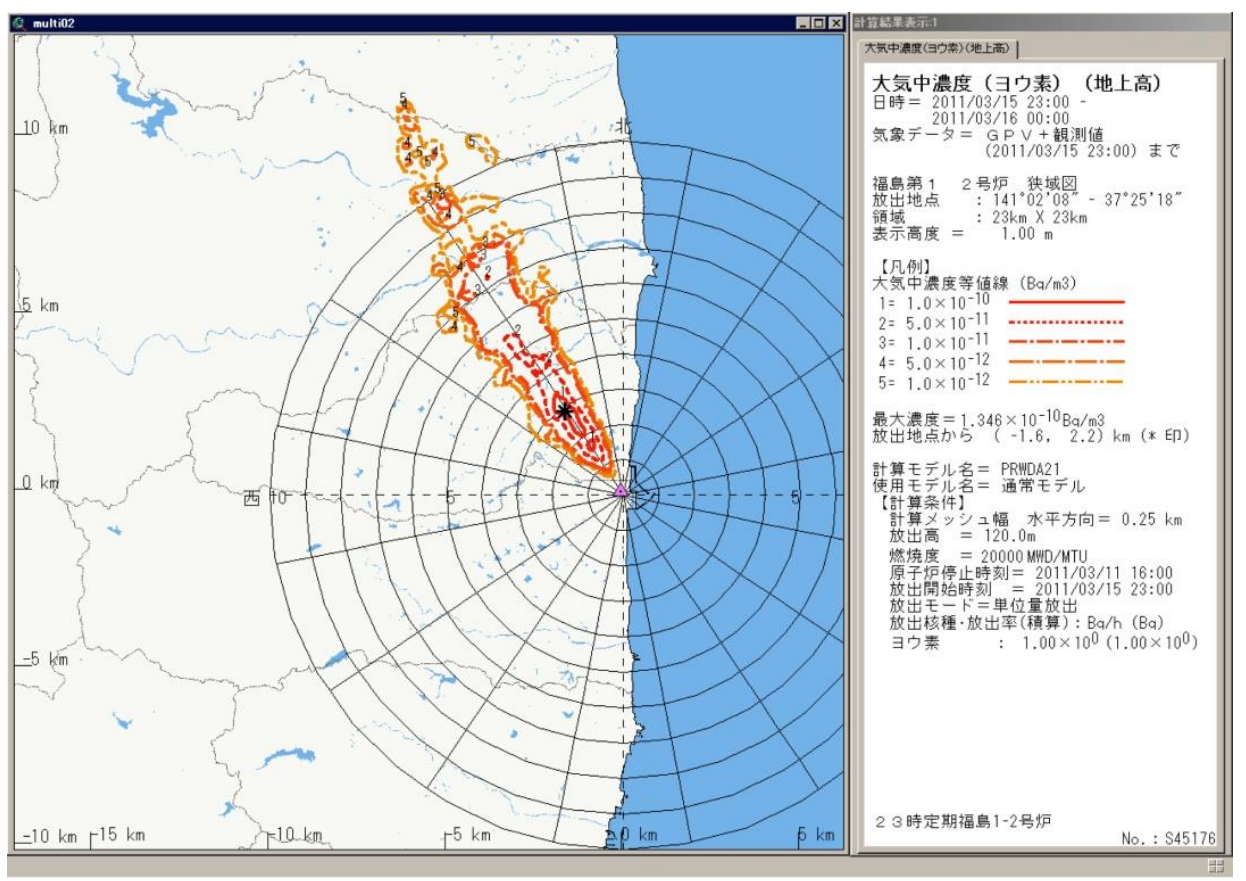

Chart 6: The Iodine release report by SPEEDI on March 15, 2011 from 11PM to midnight

Chart 6 provides SPEEDI's report on the Iodine release and Chart 7 gives rough translation of the reports in English.

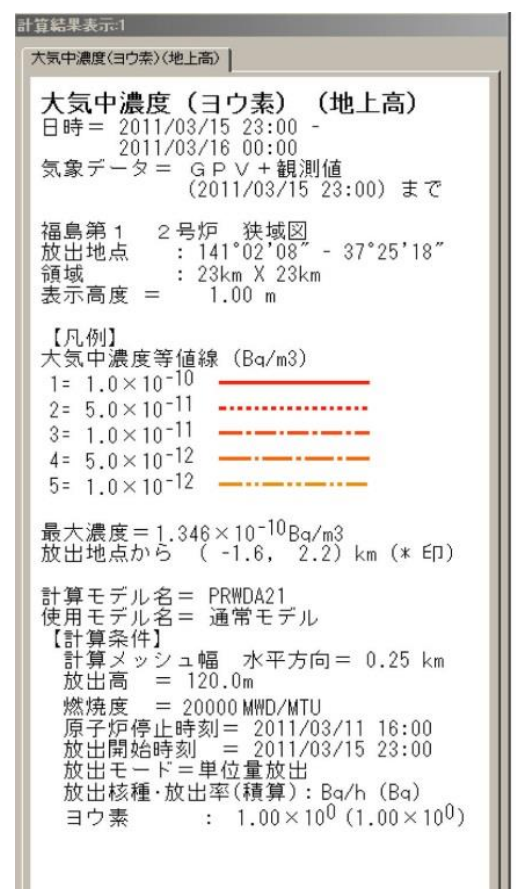

The map show an integrated value of Noble gas release on March 15, 2011 from 11PM to midnight

Fuksushima Number 2 Power Plant

Coordinates: $\quad 37^{\circ} 25^{\prime} 18^{\prime \prime} \mathrm{N}, 141^{\circ} 02^{\prime} 08^{\prime \prime} \mathrm{E}$

Radiation Grid: $23 \mathrm{~km} \times 23 \mathrm{~km}$

Site elevation 1.00 meter above sea level

Effective dosage rate for adults in $\mathrm{Bq} / \mathrm{m} 3$. (The lines show effective dose between $1 \times 10^{\wedge}-12$ to $1 \times 10^{\wedge}-10$ per hour )

The predicted maximum dose is (indicated by an asterik on the map) $1.346 \times 10^{\wedge}-10 \mathrm{~Bq} / \mathrm{m} 3$

From the release point $(-1.6,2.2) \mathrm{km}$

Calculation model name: PRWDA21

Calculation size (mesh) in horizontal direction $=.25 \mathrm{~km}$

Released amount $=1 \quad 20.0 \mathrm{~m}$

Burnup $=\quad 20,000$ MWD/MTU

Reactor shut down time: $\quad$ March 11, 2011 4PM

Release start time: March 15, 201111 PM

Release mode - unit amount release $\mathrm{Bq} / \mathrm{h}$

Release nuclear species and release rate

Noble gas (Rare gas): $1.00 \times 10^{\wedge} 0\left(1.00 \times 10^{\wedge} 0\right)$

Chart 7: Rough translation of the Iodine release report by SPEEDI 
As one can see from the reports, the amount of radiation diagnosed by SPEEDI on March 15, 2011 from $11 \mathrm{pm}$ to midnight is $1.346 \times 10^{-10}\left(\frac{\mathrm{Bq}}{\mathrm{m}^{3}}\right)$ per hour. Using relations () and (), computed values of $\sigma_{z}, \sigma_{y}$, and diagnosed values of $U=5.4, h=20$ from the reports; one can compute the source strength in $\frac{\mu g}{s e c}$ as

$$
\begin{gathered}
Q=\left(2 \pi U \sigma_{y} \sigma_{z}\right) \frac{1.346 \times 10^{-10}}{\left\{\exp \left(\frac{-(z-h)^{2}}{2 \sigma_{z}^{2}}\right)+\exp \left(\frac{-(z+h)^{2}}{2 \sigma_{z}^{2}}\right)\right\}\left\{\exp \left(\frac{-(y)^{2}}{2 \sigma_{y}^{2}}\right)\right\}} \\
=\frac{(2 \pi(5.4)(1.18064)(32.093)) \times 1.346 \times 10^{-10}}{\left\{\exp \left(\frac{-(0-20)^{2}}{2(32.093)^{2}}\right)+\exp \left(\frac{-(0+20)^{2}}{2(32.093)^{2}}\right)\right\}\left\{\exp \left(\frac{-(0)^{2}}{2(1.18064)^{2}}\right)\right\}} \\
=(1285.58689685) \frac{1.346 \times 10^{-10}}{\{0.823507867247+0.823507867247\}\{1\}} \\
=1.050627 \times 10^{-7}
\end{gathered}
$$

The computations suggest that the emission rate was $1 \mathrm{~km}$ down the plume line. Based on the meteorological conditions in the vicinity of a nuclear facility, source information that estimates the source of a radioactive release from the nuclear facility, and predetermined geographical data, the SPEEDI network system performs a radioactivity dispersion model estimates how radioactive materials disperse, their concentration in the atmosphere, and the exposure effects in human beings. These estimates are expressed in terms of contour-line data on a map (See Chart 6) showing the vicinity of the nuclear facility, and are supplied via networks (Misawa and Nagamori). 


\section{DISCUSSION}

Japan's SPEEDI network combines automatic, real time data from each of the 54 nuclear power facilities in Japan with stored data about terrains (DPNSN). When the power went out during the March 11, 2011 disaster, SPEEDI functioned correctly however, the official report indicated that the information was incomplete because a few monitoring sites were washed away, or because the data SPEEDI was producing seemed strange, but other models are provided here which all show radiation concentrating in hotspots beyond the voluntary evacuation zone.

Chart 8 illustrates evacuation zones based on the data from IAEA. US Department of Energy and National Nuclear Security Administration Data provides approximately the same evacuation zones (Chart 9). The third chart provides an insight into joint US/Japan AMS Dataset on April 29, 2011. Local citizens also used their own equipment, collecting and posting readings and data that formed a pretty good match for the government backed models.

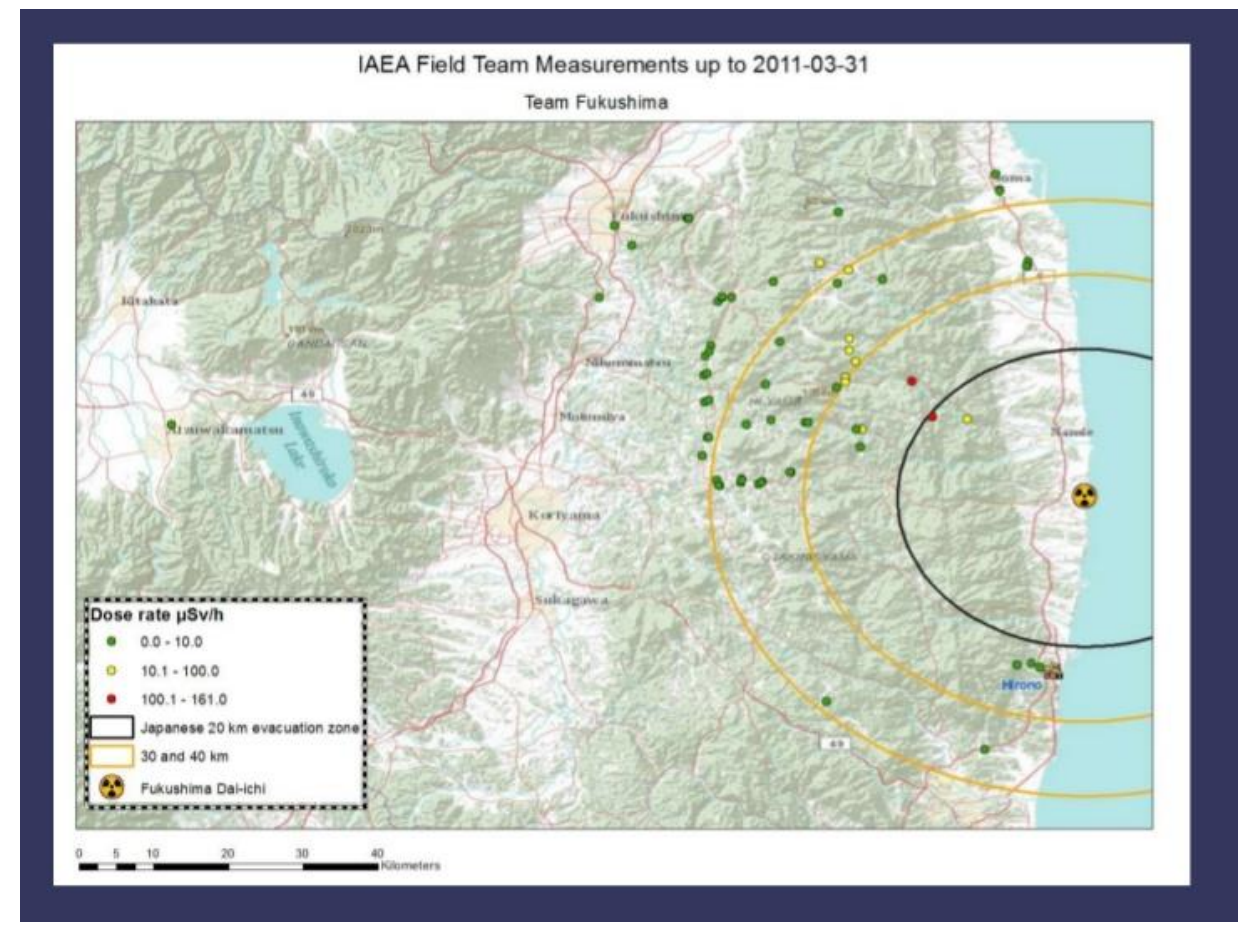


Chart 8: International Atomic Energy Agency Monitoring Database

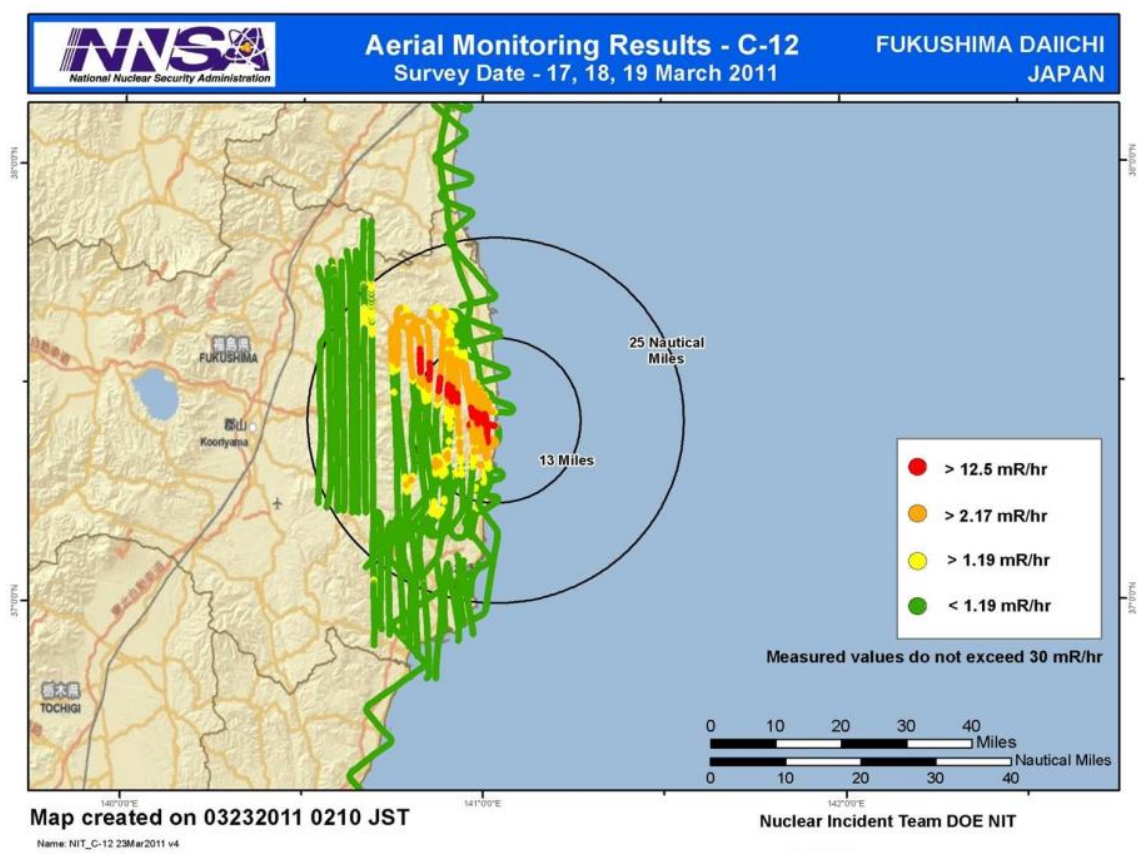

Chart 9: US Department of Energy and National Nuclear Security Administration evacuation zones in miles

\section{Joint US-Japan AMS Data}

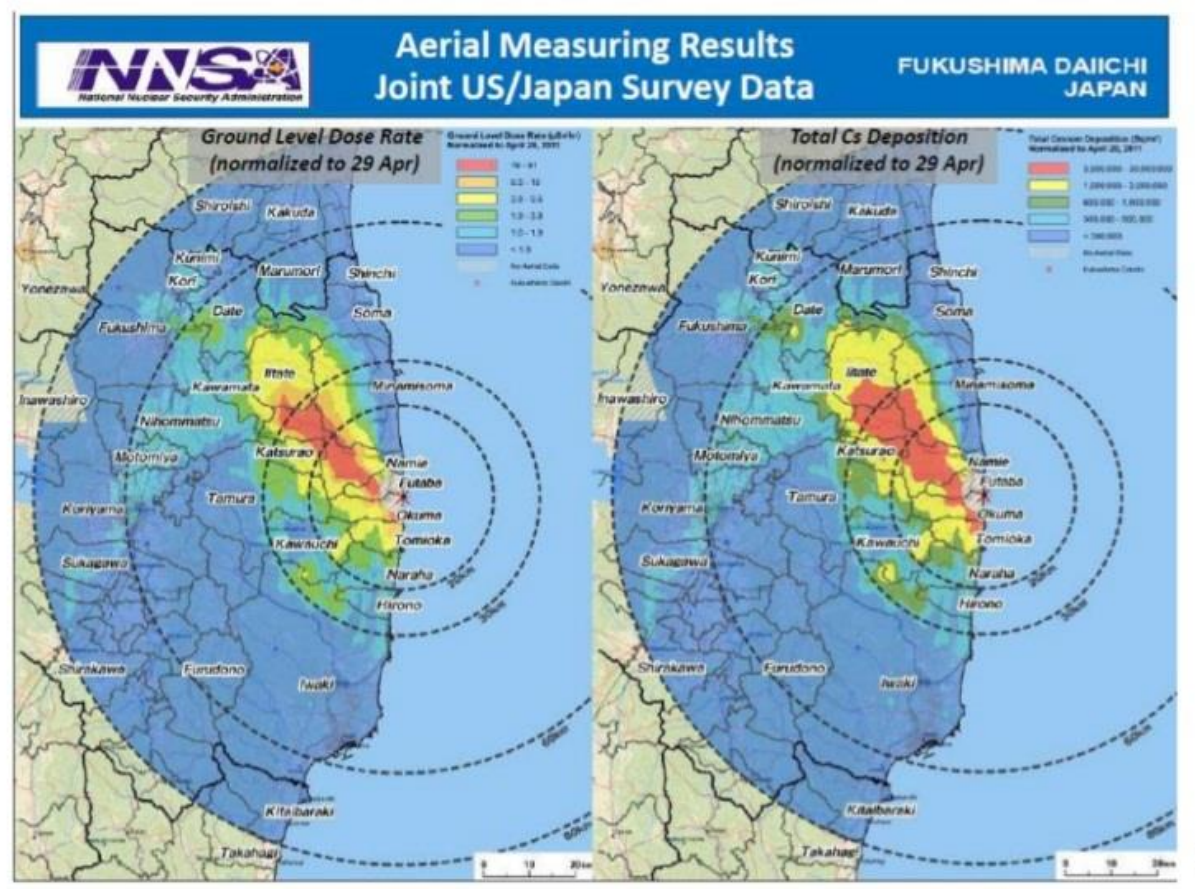


Chart 10: An insight into joint US/Japan AMS Dataset on 29 April, 2011

\section{CONCLUSION AND RECOMMENDATIONS}

There are many models that have been used to follow the radioactive particles emitted from the Fukushima incident. The Gaussian Plume model has the advantage of simple computations which can be carry out by hand, but is limited in that its predictions are valid only to within a factor of 2-3, and it can't account for curvature in the wind direction. Computer models are preferred because of the accuracy needed to make real time decisions; still they are based on the algorithms. Models tend to fall in three categories: those following Gauss (like SPEEDI), Lagrange (like FLEXpart, and HYSPLIT) which focus on particle diffusion, or Euler (like NICOIL) which looks at the mean flow of particles (National Oceanic and Atmospheric Administration). 


\section{REFERENCES}

Aldrich, Daniel P. "Post-Crisis Japanese Nuclear Policy: From Top-Down Directives to Bottom-Up Activism." AsiaPacific Issues (2012): (103): 1-12.

Anzai, Kazunori, et al. "Fukushima Daiichi Nuclear Power Plant accident: facts, environmental contamination, possible biological effects, and countermeasures." Journal of Clinical Biochemistry and Nutrition (2012): 50(1): 2-8.

Burk, David Edward. Forward model calculations for determining isotopic compositions of materials used in a radiological dispersal device. Texas A\&M University, Master of Science Thesis, 2005.

Craft, Lucille. Dissecting Disaster. Newsletter. Washington: ASEE PRISM, 2012.

Database, IAEA Fukushima Monitoring. Search and Compare Dose Rates at Monitoring Points. 2011. $\langle$ https://iec.iaea.org/fmd/compare_dose_rate_measurements.aspx $>$.

Davoine, X. and M. Bocquet. "Inverse modelling-based reconstruction of the Chernobyl source term available for long-range transport." Atmospheric Chemistry and Physics (2007): 1549-1564.

DPNSN. http://www.bousai.ne.jp/eng/. 2012. 2014.

ESS. http://www.ess.co.at/AIR-EIA/LECTURES/L001.html. 2000. 2014.

Funabashi, Yoichi and Kay Kitazawa. "Fukushima in review: A complex disaster, a disastrous response." Bulletin of the Atomic Scientists (2012): 68(2): 16-17.

Government of Japan. Cabinet Office for Disaster Management. n.d. <http://www.bousai.go.jp/>.

Gudiksen PH, PH., TF. Harvey and R. Lange. "Chernobyl source term, atmospheric dispersion, and dose estimation." Health Phys. (1989): (57): 697-706.

Hsu, Shih-Chieh, et al. "Hemispheric dispersion of radioactive plume laced with fission nuclides from the Fukushima nuclear event." Geophysical Research Letters (2012): (39): 1-6.

Larson, Ron, Robert P. Hostetler and Bruce H. Edwards. Calculus: Early Transcendental Functions, 3rd ed. Houghton Mifflin Company, 2002.

Macdonald, Robert. Theory and Objective of Air Dispersion Modelling. Wterloo: University of Waterloo, 2003.

Misawa, Makoto and Fumio Nagamori. "System for Prediction of Environmental Emergency Dose Information Network System." FUJITSU Scientific Technical Journal (2008): (44): 377-388.

MIT. MIT. n.d. <http://web.mit.edu/16.unified/www/SPRING/propulsion/notes/node66.html>.

National Oceanic and Atmospheric Administration. HYSPLIT - Hybrid Single Particle Lagrangian Integrated Trajectory Model. 30 April 2013. <http://ready.arl.noaa.gov/HYSPLIT.php>. 
Nuclear Safety Commission. Press Release. 12 March 2011. <http://www.nsr.go.jp/archive/nsc/info/110323_top_siryo.pdf>.

Onishi, Norimitsu and Martin Fackler. "Japan Held Nuclear Data, Leaving Evacuees in Peril." Newsletter. 2011.

Prevention, Centers for Disease Control and. Measuring Radiation. 25 Oct 2013. <http://www.bt.cdc.gov/radiation/measurement.asp>.

Stohl, A., et al. "Xenon-133 and caesium-137 releases into the atmosphere from the Fukushima Dai-ichi nuclear power plant: determination of the source term, atmospheric dispersion, and deposition." Atmospheric Chemistry and Physics (2012): 12: 2313-2343.

TEPCO. Impact to TEPCO's Facilities due to Miyagiken-Oki Earthquake (as of 5PM). 12 March 2011. <http://www.tepco.co.jp/en/press/corp-com/release/11031223-e.html>.

Wickert, Jonathan. An Introduction to Mechanical Engineering. CL-Engineering, 2nd Edition, 2005. 